\title{
ON THE CONTINUITY OF THE NEMITSKY OPERATOR INDUCED BY A LIPSCHITZ CONTINUOUS MAP
}

\author{
ROBERTA MUSINA
}

(Communicated by William J. Davis)

\begin{abstract}
Let $f \in \mathbb{R}^{N} \rightarrow \mathbb{R}^{k}$ be a Lipschitz continuous function, and let $\Omega$ be a bounded domain in the Euclidean space $\mathbb{R}^{n}$. For every exponent $p \in$ $\left[1,+\infty\left[\right.\right.$, the composite map $T_{f}=f \circ u$ maps the Sobolev space $W^{1, p}\left(\Omega, \mathbb{R}^{N}\right)$ into $W^{1, p}\left(\Omega, \mathbb{R}^{k}\right)$. In the scalar case, namely, when $N=1$, the operator $T_{f}$ is continuous from $W^{1, p}\left(\Omega, \mathbb{R}^{N}\right)$ into $W^{1, p}\left(\Omega, \mathbb{R}^{k}\right)$. In this paper we illustrate a counterexample to the continuity of the operator $T_{f}$ in the case where $N>1$. In the last part of the paper we give some sufficient conditions for the continuity of $T_{f}$, and we conclude with some examples.
\end{abstract}

\section{INTRODUCTION}

Let $f: \mathbb{R}^{N} \rightarrow \mathbb{R}^{k}$ be a Borel function of the Euclidean space $\mathbb{R}^{N}$ into $\mathbb{R}^{k}$. Let $u$ be a map defined on a bounded domain $\Omega$ of $\mathbb{R}^{n}$ into $\mathbb{R}^{N}$. The Nemitsky operator associated with $f$ is defined by $T u=f \circ u$, and it maps measurable functions into measurable functions.

In case $f$ is uniformly Lipschitz continuous on $\mathbb{R}^{N}$, the Nemitsky operator $T$ maps the Sobolev space $W^{1, p}\left(\Omega, \mathbb{R}^{N}\right)$ into $W^{1, p}\left(\Omega, \mathbb{R}^{k}\right)$, and

$$
\|f \circ u-f(0)\|_{W^{1, p}\left(\Omega, \mathbb{R}^{k}\right)} \leq \operatorname{Lip}(f)\|u\|_{W^{1, p}\left(\Omega, \mathbb{R}^{N}\right)},
$$

where $\operatorname{Lip}(f)$ is the Lipschitz constant of $f$. In this introduction we restrict our attention to the case $1<p<\infty$. Using (0.1) and the continuity of $f$, we immediately get that the operator $T$ is sequentially continuous with respect to the weak topologies in the domain and in the target space. In this paper we investigate the continuity properties of the operator $T$ with respect to the $W^{1 . p}$-norms. For a reference to this problem see Steffen [10, p. 104].

When $f$ has continuous first-order partial derivatives, then the chain rule holds:

$$
\nabla(f \circ u)=\nabla f(u) \cdot \nabla u \text { almost everywhere on } \Omega,
$$

Received by the editors August 20, 1989 and, in revised form, February 20, 1990.

1980 Mathematics Subject Classification (1985 Revision). Primary 46E35. 
and, since the map $\nabla f(u)$ is continuous in $u$, the continuity of $T$ follows immediately. Clearly enough, when $f$ is not a $C^{1}$ function on $\mathbb{R}^{N}$, the above argument can not be used all over again. Nevertheless, in the scalar case (namely, when $N=1)$, the chain rule (0.2) still holds, and the operator $T$ is continuous from $W^{1, p}(\Omega, \mathbb{R})$ into $W^{1, p}\left(\Omega, \mathbb{R}^{k}\right)$.

Theorem 0.1. For every Lipschitz continuous function $f: \mathbb{R} \rightarrow \mathbb{R}^{k}$, the associated Nemitsky operator is continuous from $W^{1, p}(\Omega, \mathbb{R})$ into $W^{1, p}\left(\Omega, \mathbb{R}^{k}\right)$.

The chain rule $(0.2)$ and Theorem 0.1 were first proved by Marcus and Mizel [7], [8]. We refer also to [3] and [4] for different proofs.

When $u$ is a vector-valued function, the chain rule $(0.2)$ is no longer true. Recently, Ambrosio and Dal Maso have proved [1] a general chain rule for distributional derivatives of the composite map $f \circ u$, where $f$ is Lipschitz continuous and $u$ has bounded variation (see Lemma 2.1 below). When $u \in$ $W^{1, p}\left(\Omega, \mathbb{R}^{N}\right)$, from this chain rule we infer that in the general case the derivatives of the composite function $f \circ u$ can be expressed by means of a Borel function of $u$ and $\nabla u$ which is neither linear nor continuous in $\nabla u$. For this reason in the general case a continuity result for the operator $T$ is completely hopeless.

In $\S 1$ we shall illustrate a counterexample which shows that, in general, the operator $T$ is not continuous if $N>1$ and $f$ is only Lipschitz continuous.

In $\S 2$ we look for sufficient conditions on $f$ which assure the continuity of the associated Nemitsky operator. The continuity criterion stated here applies, for instance, to Lipschitz continuous maps on $\mathbb{R}^{N}$ which have continuous first order derivatives outside a closed singular set $S$ having empty interior.

Our results involve a suitable notion of a tangent space $T_{S}$ to the singular set $S$. More precisely, for every point $u_{0}$ in $S$, we define

$$
T_{S}\left(u_{0}\right)=\left\{\tau \in \mathbb{R}^{N} \mid \lim _{h \rightarrow 0} \frac{d_{S}\left(u_{0}+h \tau\right)}{h}=0\right\},
$$

where $d_{S}(\cdot)$ denotes the Euclidean distance from the set $S$. Roughly speaking, in order to have the continuity of the Nemitsky operator associated with a Lipschitz continuous map $f \in C^{1}\left(\mathbb{R}^{N} \backslash S, \mathbb{R}^{k}\right)$, we only need a sort of "good behavior" of $\nabla f(u) \cdot \tau$, when $u \notin S$ and $u \rightarrow u_{0} \in S$, for every tangent direction $\tau$ to $S$ at the point $u_{0}$.

The class of maps considered above attracts our interest essentially for two reasons. First, Lipschitz continuous maps which are smooth off a small singular set are quite frequently in use (see the examples in $\S 3$ ). Second, the map $f$ of the counterexample in $\S 1$ belongs to this same class. From this viewpoint, the criterion we present here indicates what is really responsible for the continuity of the Nemitsky operator associated with a Lipschitz continuous map on $\mathbb{R}^{N}$.

We conclude the paper with a few additional examples, in $\S 3$.

Notation. For $\Omega \subset \mathbb{R}^{n}$ open and bounded and for $p \in[1, \infty[$, we denote by $W^{1, p}\left(\Omega, \mathbb{R}^{N}\right)$ the space of $N$-tuples of functions in $W^{1, p}(\Omega, \mathbb{R})$, endowed 
with the usual weak and strong topologies. If $C$ is a closed subset of $\mathbb{R}^{N}$, the class

$$
W^{1, p}(\Omega, C):=\left\{u \in W^{1, p}\left(\Omega, \mathbb{R}^{N}\right) \mid u(x) \in C \text { for a.e. } x \in \Omega\right\}
$$

is closed in the weak topology of $W^{1, p}\left(\Omega, \mathbb{R}^{N}\right)$.

Let $E$ be a subset of $\mathbb{R}^{N}$. We denote by $\operatorname{Lip}\left(E, \mathbb{R}^{k}\right)$ the class of uniformly Lipschitz continuous maps from $E$ into $\mathbb{R}^{k}$, and for every map $f \in$ $\operatorname{Lip}\left(E, \mathbb{R}^{k}\right)$, we denote by $\operatorname{Lip}(f)$ the Lipschitz constant of $f$ on $E$.

For any open subset $A$ of $\mathbb{R}^{N}$, we shall denote by $C^{1}\left(\bar{A}, \mathbb{R}^{k}\right)$ the class of maps $f: A \rightarrow \mathbb{R}^{k}$ which are continuously differentiable on $A$ such that the maps $f$ and its gradient $\nabla f$ have continuous extensions $f, \nabla f$ to the closure $\bar{A}$ of $A$.

In what follows, $g$ will always denote a Lipschitz continuous map defined on $\mathbb{R}^{N}$, and $T_{g} u:=g \circ u$ is the associated Nemitsky operator. Then the operator $T_{g}$ maps the Sobolev space $W^{1, p}\left(\Omega, \mathbb{R}^{N}\right)$ into $W^{1, p}\left(\Omega, \mathbb{R}^{k}\right)$. This fact is well known (see for example [9]), and it can easily be proved by approximating $g$ with a sequence of Lipschitz functions of class $C^{1}$. For a given Lipschitz continuous function $f$ defined on a subset $E$ of $\mathbb{R}^{N}$, the associated Nemitsky operator maps $W^{1, p}(\Omega, \bar{E})$ into $W^{1, p}\left(\Omega, \mathbb{R}^{k}\right)$. To see this, we take an extension $g \in \operatorname{Lip}\left(\mathbb{R}^{N}, \mathbb{R}^{k}\right)$ of $f$. Since $T_{g}$ maps $W^{1, p}\left(\Omega, \mathbb{R}^{N}\right)$ into $W^{1, p}\left(\Omega, \mathbb{R}^{k}\right)$, and since $T_{g_{\mid W^{1, p}(\Omega, \bar{E})}}=T_{f}$, the conclusion easily follows.

\section{A COUNTEREXAMPLE}

The main ideas for the construction of the counterexample are due to Gianni Dal Maso.

We shall construct a Lipschitz continuous map $f: \mathbb{R}^{2} \rightarrow \mathbb{R}$ whose associated superposition operator $T_{f}$ is not continuous on Sobolev spaces. Actually, the map $f$ we are going to construct could not be said to be too irregular. On the contrary, it has some nice properties, such as the following:

(a) $f$ is everywhere differentiable;

(b) $f$ is continuously differentiable outside the singular set $S=\{0\} \times \mathbb{R}$; and

(c) $f(z)=0$ and $\nabla f(z)=0$ for every point $z$ in the singular set.

The only defects of $\nabla f$ arise along the tangent directions to the singular set; that is the partial derivatives of $f$ along the tangent directions to $S$ are not continuous on $\mathbb{R}^{2}$. Actually, this is the only true obstruction to the continuity of $T_{f}$. In fact, as we shall see in $\S 3$, in the class $\mathscr{L}$ of all Lipschitz continuous maps $g$ on $\mathbb{R}^{2}$ which are continuously differentiable outside the set $S$ such that $g=0$ on $S$, the condition

$$
\lim _{\substack{x \rightarrow 0 \\ x \neq 0}} \frac{\partial}{\partial y} g(x, y)=0 \quad \forall y \in \mathbb{R}
$$


is necessary and sufficient for the continuity of the Nemitsky operator $T_{g}$ (see Example 3.1).

Let us now try to describe the shape of the map $f$ in the counterexample. For $x \neq 0$ fixed, the map $y \rightarrow f(x, y)$ oscillates with a period $t_{x}$ which goes to zero as $x \rightarrow 0$. Since $f$ has to be Lipschitz continuous, the maximum variation of the map $y \rightarrow f(x, y)$ must be of the same order as $t_{x}$. About the dependence on the variable $x$, we shall define $f$ in such a way that for every $y \in \mathbb{R}$ fixed, the zeros of the map $x \rightarrow f(x, y)$ accumulate at 0 . Since $f$ has to be Lipschitz continuous, the amplitude of oscillations of $f(\cdot, y)$ is forced to go to 0 as $x \rightarrow 0$. Moreover, the dependence on the variables $x$ and $y$ has to be balanced in such a way that the resulting function $f$ does not verify (1.1).

For every positive integer $h$, we set $\left.I_{h}=\right] \frac{1}{h+1}, \frac{1}{h}$ [, and we define the sequences $a_{h}=2 h(h+1)$ and $b_{h}=2 h+1$. These two sequences are chosen in such a way that $b_{h} / a_{h}$ is the barycentre of the interval $I_{h}$. Now we fix any function $\varphi \in C_{0}^{\infty}(\mathbb{R})$ such that

$$
\operatorname{supp} \varphi \subseteq]-1,1[, \varphi(0)=1 .
$$

The map $f$ is defined by:

$$
f(x, y)= \begin{cases}\frac{1}{a_{h}} \varphi\left(a_{h}|x|-b_{h}\right) \sin \left(a_{h} y\right) & \text { if }|x| \in \bar{I}_{h} \\ 0 & \text { if } x=0 .\end{cases}
$$

Notice that, if $\varphi\left(a_{h}|x|-b_{h}\right) \neq 0$ for a point $x \neq 0$ and for a positive integer $h$, then we get $\left(b_{h}-1\right) a_{h}^{-1}<|x|<\left(b_{h}+1\right) a_{h}^{-1}$, that is, $|x| \in I_{h}$. This shows that the map $f$ is well defined on $\mathbb{R}^{2}$ and is of class $C^{\infty}$ outside the set $\{0\} \times \mathbb{R}$. We notice that

$$
|f(x, y)| \leq|x|^{2} \quad \forall(x, y) \in \mathbb{R}^{2},
$$

and hence we can easily check the continuity of $f$ on $\mathbb{R}^{2}$. Moreover, for every $x \neq 0$ and for every $y \in \mathbb{R}$ it results

$$
\begin{aligned}
\left|\frac{\partial}{\partial y} f(x, y)\right| & \leq \sum_{h}\left|\varphi\left(a_{h}|x|-b_{h}\right) \cos \left(a_{h} y\right)\right| \leq\|\varphi\|_{L^{\infty}}, \\
\left|\frac{\partial}{\partial x} f(x, y)\right| & \leq \sum_{h}\left|\varphi^{\prime}\left(a_{h}|x|-b_{h}\right) \sin \left(a_{h} y\right)\right| \leq\left\|\varphi^{\prime}\right\|_{L^{\infty}} .
\end{aligned}
$$

Since the derivatives of $f$ are bounded, we infer that $f$ is Lipschitz continuous on $\mathbb{R}^{2}$, and $\operatorname{Lip}(f) \leq\|\varphi\|_{C^{1}}$. In addition, we notice that $f$ is differentiable at every point $(0, y)$, and by $(1.2)$ we get $\frac{\partial}{\partial x} f(0, y)=0$ for every $y \in \mathbb{R}$.

In order to show that $T_{f}$ is not continuous on Sobolev spaces, we consider the sequence $u_{h}$ in $C^{\infty}\left(\mathbb{R}, \mathbb{R}^{2}\right)$ defined by

$$
u_{h}(t)=\left(b_{h} / a_{h}, t\right) \text {. }
$$

Notice that $u_{h} \rightarrow u$ in $C^{\infty}\left(\mathbb{R}, \mathbb{R}^{2}\right)$ where $u(t)=(0, t)$, and in particular 
$u_{h} \rightarrow u$ in $H_{\text {loc }}^{1, p}\left(\mathbb{R}, \mathbb{R}^{2}\right)$ for every exponent $p$. On the other hand, $\left(f \circ u_{h}\right)(t)=$ $\frac{1}{a_{h}} \sin \left(a_{h} t\right) \rightarrow 0$ pointwise, and

$$
\frac{d}{d t}\left(f \circ u_{h}\right)(t)=\cos \left(a_{h} t\right) .
$$

Since $a_{h} \rightarrow+\infty$, we have that the sequence $\frac{d}{d t}\left(f \circ u_{h}\right)$ does not have almosteverywhere-convergent subsequences, and hence it does not converge in $L_{\text {loc }}^{p}\left(\mathbb{R}, \mathbb{R}^{2}\right)$.

This completes the proof of the counterexample.

\section{SOME SUFFICIENT CONDITIONS}

In the following, we shall look for some simple continuity criterion for the operator $T_{f}$ associated with a Lipschitz continuous map defined on $\mathbb{R}^{N}$. The main tool will be the following chain rule, which is due to Ambrosio and Dal Maso [1].

Lemma 2.1. Let $g \in \operatorname{Lip}\left(\mathbb{R}^{N}, \mathbb{R}^{k}\right)$, and let $w \in W^{1, p}\left(\Omega, \mathbb{R}^{N}\right)$. Then, for almost every $x \in \Omega$, the restriction of $g$ to the affine space $A_{w, \nabla w}:=\left\{y=\left(y^{1}, \ldots, y^{N}\right) \in \mathbb{R}^{N} \mid y^{i}=w^{i}(x)+\left\langle\nabla w^{i}(x), z\right\rangle\right.$ for some $\left.z \in \mathbb{R}^{n}\right\}$ is differentiable at $w(x)$, and for every coordinate $x_{j}$ in $\mathbb{R}^{n}$ we have that

$$
\frac{\partial}{\partial x_{j}}(g \circ w)(x)=\nabla\left(\left.g\right|_{A_{w, \nabla w}}\right)(w(x)) \cdot \frac{\partial}{\partial x_{j}} w(x) \text { a.e. in } \Omega \text {. }
$$

We shall first consider the case of a Lipschitz continuous map $f: E \rightarrow \mathbb{R}^{k}$ defined on a subset $E$ of $\mathbb{R}^{N}$. The map $f$ admits a unique Lipschitz continuous extension $f: \bar{E} \rightarrow \mathbb{R}^{k}$, but in the following it will be often convenient to make a distinction between the map $f: E \rightarrow \mathbb{R}^{k}$ and its extension $f: \bar{E} \rightarrow \mathbb{R}^{k}$.

Let $g: \mathbb{R}^{N} \rightarrow \mathbb{R}^{k}$ be any Lipschitzian extension of $f$ to $\mathbb{R}^{N}$ (see, for example [6, p. 202 and Theorem 2.10.43]). By Rademacher's Theorem, the map $g$ is differentiable almost everywhere on $\mathbb{R}^{N}$. In particular, for almost every $u \in \mathbb{R}^{N}$ and for every $\tau \in \mathbb{R}^{N}$, there exists the partial derivative of $g$ at $u$ in the direction $\tau$, i.e., there exists

$$
D g(u, \tau)=\lim _{h \rightarrow 0} \frac{g(u+h \tau)-g(u)}{h} .
$$

We denote by $\mathscr{D}(D g)$ the set of pairs $(u, \tau) \in \mathbb{R}^{N} \times \mathbb{R}^{N}$ for which the limit in (2.2) exists. In this way we have defined a map

$$
D g: \mathscr{D}(D g) \subseteq \mathbb{R}^{N} \times \mathbb{R}^{N} \rightarrow \mathbb{R}^{k}
$$

Let $u_{0}$ be any point in the interior of $E$, and let $\tau \in \mathbb{R}^{N}$. If $\left(u_{0}, \tau\right) \in \mathscr{D}(D g)$, then the map $f$ is differentiable at $u_{0}$ along the direction $\tau$, and the vector

$$
D f\left(u_{0}, \tau\right)=\lim _{h \rightarrow 0} \frac{f\left(u_{0}+h \tau\right)-f\left(u_{0}\right)}{h}
$$


is equal to $D g\left(u_{0}, \tau\right)$. Our aim is to define $D f\left(u_{0}, \tau\right)$ for points $u_{0} \in \partial E$ and for some privileged directions $\tau$ which will be described as the tangent directions to $\partial E$ at $u_{0}$.

We first introduce a convenient notion of a tangent space to an arbitrary set $S$ in $\mathbb{R}^{N}$. For any subset $S \subseteq \mathbb{R}^{N}$, we denote by $d_{S}$ the Lipschitz continuous function

$$
d_{S}(w)=\operatorname{Inf}_{u \in S}|u-w| .
$$

Suppose now that $S$ is closed, and fix a point $u_{0}$ in $S$. We shall say that a vector $\tau \in \mathbb{R}^{N}$ is a tangent vector to $S$ at $u_{0}$ if

$$
\limsup _{h \rightarrow 0} \frac{d_{S}\left(u_{0}+h \tau\right)}{h}=0 .
$$

The set of all tangent vectors to $S$ at $u_{0}$ will be called the tangent space to $S$ at $u_{0}$, and it will be denoted by $T_{S}\left(u_{0}\right)$

$$
T_{S}\left(u_{0}\right)=\left\{\tau \in \mathbb{R}^{N} \mid \lim _{h \rightarrow 0} \frac{d_{S}\left(u_{0}+h \tau\right)}{h}=0\right\} .
$$

We notice that $T_{S}\left(u_{0}\right)$ is a closed cone, and it is symmetric with respect to the origin. Looking at $T_{S}$ as a multivalued map $T_{S}: S \rightarrow \mathscr{P}\left(\mathbb{R}^{N}\right)$, it is natural to consider its graph

$$
G\left(T_{S}\right)=\left\{(u, \tau) \in S \times \mathbb{R}^{N} \mid \tau \in T_{S}(u)\right\} .
$$

In order to justify this definition, we observe that $T_{S}\left(u_{0}\right)$ coincides with the tangent space of differential geometry if $S$ is a $C^{1}$ manifold without boundary; if $\partial S$ is not empty and $u_{0} \in \partial S$, then $T_{S}\left(u_{0}\right)$ is the tangent space to $\partial S$ at $u_{0}$ in the sense of differential geometry. For further comments about the concept of tangent space and of tangent cone we refer to [2, Chapter 7, p. 492].

Remark 2.2.

(i) $T_{S}\left(u_{0}\right) \subseteq$ closure of $\bigcup_{h \neq 0} \frac{S-u_{0}}{h}$.

(ii) If $u_{0}$ belongs to the interior of $S$, then $T_{S}\left(u_{0}\right)=\mathbb{R}^{N}$.

(iii) If $u_{0}$ is an isolated point in $S$, then $T_{S}\left(u_{0}\right)=\{0\}$.

We notice that the condition: $T_{S}\left(u_{0}\right) \neq\{0\}$, forces the set $S$ to be a little regular at the point $u_{0} \in S$, since for every nonzero tangent vector $\tau \in T_{S}\left(u_{0}\right)$, we have that the restriction of the function $d_{S}$ to the line $\left\{u_{0}+\lambda \tau\right\}_{\lambda \in \mathbb{R}}$ is differentiable at $\lambda=0$.

Now we go back to the map $f \in \operatorname{Lip}\left(E, \mathbb{R}^{k}\right)$ and to its extension $g \in$ $\operatorname{Lip}\left(\mathbb{R}^{N}, \mathbb{R}^{k}\right)$. We set $\mathscr{D}(D f)=\mathscr{D}(D g) \cap\left[\left(E^{0} \times \mathbb{R}^{N}\right) \cup G\left(T_{\partial E}\right)\right]$; that is,

$$
\mathscr{D}(D f)=\left\{(u, \tau) \in \bar{E} \times \mathbb{R}^{N} \mid u \in \stackrel{0}{E} \text { or } \tau \in T_{\partial E}(u)\right\} \cap \mathscr{D}(D g) .
$$

For every pair $(u, \tau) \in \mathscr{D}(D f)$, we define

$$
D f(u, \tau)=D g(u, \tau) .
$$


In the next lemma we show that the above definitions do not depend on the choice of the extension $g$, and hence for every $(u, \tau) \in \mathscr{D}(D f)$ the vector $D f(u, \tau)$ can be seen as the partial derivative of $f$ at the point $u$ in the direction $\tau$.

Lemma 2.3. The set $\mathscr{D}(D f)$ and the map $D f: \mathscr{D}(D f) \rightarrow \mathbb{R}^{k}$ do not depend on the extension $g$ of $f$. In addition, for every $u \in \bar{E}$ the set $\left\{\tau \in \mathbb{R}^{N} \mid(u, \tau) \in\right.$ $\mathscr{D}(D f)\}$ is closed, and the map $\tau \rightarrow D f(u, \tau)$ is continuous at every point of its domain.

Proof. The map $f$ extends in a unique way to the closure of $E$. We denote again by $f$ the extension. Let $(u, \tau)$ be any fixed pair in $\left(\stackrel{0}{E} \times \mathbb{R}^{N}\right) \cup G\left(T_{\partial E}\right)$. We can suppose that $u \in \partial E$ and $\tau \in T_{\partial E}(u)$. By Remark 2.2(i), we have that for every sequence $h_{n} \rightarrow 0$, there exists a sequence $\tau_{n} \rightarrow \tau$ such that $u+h_{n} \tau_{n} \in \partial E$. From $g\left(u+h_{n} \tau_{n}\right)=f\left(u+h_{n} \tau_{n}\right)$, we find

$$
\left|\frac{g\left(u+h_{n} \tau\right)-g(u)}{h_{n}}-\frac{f\left(u+h_{n} \tau_{n}\right)-f(u)}{h_{n}}\right| \leq \operatorname{Lip}(g)\left|\tau-\tau_{n}\right| .
$$

This proves that

$$
D f(u, \tau)=\lim _{\substack{h \rightarrow 0, \tau^{\prime} \rightarrow \tau \\ u+h \tau^{\prime} \in \partial E}} \frac{f\left(u+h \tau^{\prime}\right)-f(u)}{h}
$$

(whenever this limit exists), and completes the proof of the first part of the lemma. In order to prove the second part, it suffices to note that for every $u, \tau, \tau^{\prime} \in \mathbb{R}^{N}$ and for every $h \neq 0$,

$$
\left|\frac{g(u+h \tau)-g(u)}{h}-\frac{g\left(u+h \tau^{\prime}\right)-g(u)}{h}\right| \leq \operatorname{Lip}(g)\left|\tau-\tau^{\prime}\right|,
$$

where $\operatorname{Lip}(g)$ denotes the Lipschitz constant of $g$ on $\mathbb{R}^{N}$. Hence it follows that, for every fixed point $u \in \mathbb{R}^{N}$, the set $\left\{\tau \in \mathbb{R}^{N} \mid(u, \tau) \in \mathscr{D}(D g)\right\}$ is closed and the map $\tau \rightarrow D g(u, \tau)$ is continuous at every point of its domain. The conclusion readily follows from the definition of $D f$ and of $\mathscr{D}(D f)$.

In the following, we shall use the lemma by Ambrosio and Dal Maso in order to relate the derivatives of $W^{1, p}$-functions to the notions just introduced.

Lemma 2.4. Let $w$ be a function in $W^{1, p}\left(\Omega, \mathbb{R}^{N}\right)$ and let $S$ be a closed subset of $\mathbb{R}^{N}$.

Then for every $j=1, \ldots, n$ we have

$$
\frac{\partial}{\partial x_{j}} w(x) \in T_{S}(w(x)) \quad \text { for almost every } x \in w^{-1}(S) .
$$

Proof. We define $v(x):=d_{S}(w(x))$. Then $v \in W_{\text {loc }}^{1, p}\left(\Omega, \mathbb{R}^{N}\right)$ since $d_{S}$ is Lipschitz continuous on $\mathbb{R}^{N}$. By a result on Sobolev spaces (for example [6], Theorem 3.1.6), we have that $\nabla v(x)=0$ almost everywhere on $v^{-1}(\{0\})$, 
and since $w^{-1}(S) \subseteq v^{-1}(\{0\})$, the lemma follows by applying Lemma 2.1 with $f=d_{S}$.

From Lemma 2.1 and Lemma 2.4 we immediately get the next corollary:

Corollary 2.5. Let $E$ be a subset of $\mathbb{R}^{N}$, and let $f \in \operatorname{Lip}\left(E, \mathbb{R}^{k}\right)$ be a given function. Then for every map $u \in W^{1, p}\left(\Omega, \mathbb{R}^{N}\right)$, there exists a subset $\Omega_{u}$ of $\Omega$ having zero Lebesgue measure, such that

$$
\begin{gathered}
\left(u(x), \frac{\partial}{\partial x_{j}} u(x)\right) \in \mathscr{D}(D f) \quad \text { for every } x \in \Omega \backslash \Omega_{u}, \\
\frac{\partial}{\partial x_{j}}(f \circ u)(x)=D f\left(u(x), \frac{\partial}{\partial x_{j}} u(x)\right) \quad \text { for every } x \in \Omega \backslash \Omega_{u}
\end{gathered}
$$

and for every coordinate $x_{j}$ in $\mathbb{R}^{n}$.

We are now in position to state the continuity criterion. Let $f \in \operatorname{Lip}\left(E, \mathbb{R}^{k}\right)$ be a given function defined on a subset $E$ of $\mathbb{R}^{N}$. For every finite partition $\left(E_{i}\right)_{i=1, \ldots, s}$ of $E$ into subsets $E_{i}$, we set

$$
f_{i}=\left.f\right|_{E_{i}} \text {. }
$$

Proposition 2.6. Let $f \in \operatorname{Lip}\left(E, \mathbb{R}^{k}\right)$ be a given function and let $\left(E_{i}\right)_{i=1, \ldots, s}$ be a finite partition of $E$. Assume that for every index $i$ the map $D f_{i}: \mathscr{D}\left(D f_{i}\right) \rightarrow \mathbb{R}^{k}$ is continuous at every point of its domain $\mathscr{D}\left(D f_{i}\right) \subseteq\left(\stackrel{0}{E}_{i} \times \mathbb{R}^{N}\right) \cup G\left(T_{\partial E_{i}}\right)$. Then the Nemitsky operator associated with $f$ is continuous from $W^{1, p}(\Omega, \bar{E})$ into $W^{1, p}\left(\Omega, \mathbb{R}^{k}\right)$.

Proof. We fix a sequence $\left(u_{n}\right)_{n}$ in $W^{1, p}(\Omega, \bar{E})$ such that $u_{n} \rightarrow u$ strongly in $W^{1, p}$. In order to prove the continuity of $T_{f}$, we have to show that $f \circ u_{n} \rightarrow$ $f \circ u$ in $W^{1, p}\left(\Omega, \mathbb{R}^{k}\right)$. Actually, it suffices to prove that (for a subsequence)

$$
\nabla\left(f \circ u_{n}\right) \rightarrow \nabla(f \circ u) \text { almost everywhere in } \Omega \text {. }
$$

In fact, using the Lipschitz continuity assumption on $f$, and using the generalized Lebesgue's Theorem, we first get $f \circ u_{n} \rightarrow f \circ u$ in $L^{p}\left(\Omega, \mathbb{R}^{k}\right)$. Moreover, we can extend $f$ to $\mathbb{R}^{N}$ to get

$$
\left|\nabla\left(f \circ u_{n}\right)\right| \leq \operatorname{Lip}(f)\left|\nabla u_{n}\right| \quad \text { a.e. on } \Omega
$$

(compare with (2.1)), and hence the proof of Proposition 2.6 will follow from (2.4) and from Lebesgue's Theorem.

Since $u_{n} \rightarrow u$ in $W^{1, p}\left(\Omega, \mathbb{R}^{N}\right)$, we can find a null subset $\Omega_{0}$ of $\Omega$ such that

$$
\begin{gathered}
u_{n}(x) \rightarrow u(x) \quad \forall x \in \Omega \backslash \Omega_{0}, \\
\frac{\partial}{\partial x_{j}} u_{n}(x) \rightarrow \frac{\partial}{\partial x_{j}} u(x) \quad \forall x \in \Omega \backslash \Omega_{0} \text { and } \forall j=1, \ldots, n .
\end{gathered}
$$


For simplicity, we first assume that $s=1$; that is, we assume that the map $D f$ is continuous on its domain. We can assume that the null set $\Omega_{0}$ contains the sets $\Omega_{u}, \Omega_{u_{n}}$ for every $n \geq 1$ (compare with Corollary 2.5). We first get that, for every $x \in \Omega \backslash \Omega_{0}$ and for every $j=1, \ldots, n$, the sequence $\left(u_{n}(x), \frac{\partial}{\partial x_{j}} u_{n}(x)\right)$ belongs to $\mathscr{D}(D f)$ and converges to $\left(u(x), \frac{\partial}{\partial x_{j}} u(x)\right) \in \mathscr{D}(D f)$ by $(2.5)$ and (2.6). Thus, the assumption on $f$ gives

$$
D f\left(u_{n}(x), \frac{\partial}{\partial x_{j}} u_{n}(x)\right) \rightarrow D f\left(u(x), \frac{\partial}{\partial x_{j}} u(x)\right) \quad \text { for every } j=1, \ldots, n,
$$

which is equivalent to

$$
\frac{\partial}{\partial x_{j}}\left(f \circ u_{n}\right)(x) \rightarrow \frac{\partial}{\partial x_{j}}(f \circ u)(x) \quad \text { for every } j=1, \ldots, n,
$$

by Corollary 2.5. Since $x$ is an arbitrary point in $\Omega \backslash \Omega_{0}$, this proves the assertion.

Now we prove (2.4) in the general case. We fix a coordinate $x_{j}$ in $\mathbb{R}^{n}$. By a result on Sobolev spaces, we get that for every map $w \in W^{1, p}(\Omega, \bar{E})$ and for every index $i$,

$$
\frac{\partial}{\partial x_{j}}(f \circ w)(x)=\frac{\partial}{\partial x_{j}}\left(f_{i} \circ w\right)(x) \quad \text { for a.e. } x \in w^{-1}\left(\bar{E}_{i}\right) \text {. }
$$

Since the sets $E_{i}$ cover $E$, by using (2.7), the continuity of $D f_{i}$, and the arguments in the first part of the proof, we infer that for almost every $x \in \Omega$, there exist an index $i_{x}$ and a subsequence $u_{n_{k}}$, such that $u_{n_{k}}(x) \in \bar{E}_{i_{x}}$ for every $k$, and

$$
\frac{\partial}{\partial x_{j}}\left(f \circ u_{n_{k}}\right)(x)=\frac{\partial}{\partial x_{j}}\left(f_{i_{x}} \circ u_{n_{k}}\right)(x) \rightarrow \frac{\partial}{\partial x_{j}}(f \circ u)(x) .
$$

Finally, since the limit in (2.8) does not depend on the subsequence $u_{n_{k}}$, we can conclude that (2.4) holds, and the proposition is proved.

It can be proved that subsets $E_{0}$ of $E$ having zero 1-dimensional Lebesgue measure can be neglected. This easily follows using the fact that, for every map $u \in W^{1, p}(\Omega, \bar{E}), \nabla u=0$ a.e. on $u^{-1}\left(E_{0}\right)$.

Remark 2.7. Let $E$ be an open subset of $\mathbb{R}^{N}$, and let $f$ be a Lipschitz continuous function on $E$ with $f \in C^{1}\left(\bar{E}, \mathbb{R}^{k}\right)$. By definition, the gradient of $f$ has a continuous extension to the closure of $E$. In case the boundary of $E$ is smooth enough, it is clear that $\mathscr{D}(D f)=\left(\stackrel{0}{E} \times \mathbb{R}^{N}\right) \cup G\left(T_{\partial E}\right)$, and

$$
\nabla f(u) \cdot \tau=D f(u, \tau) \text { for every }(u, \tau) \in G\left(T_{\partial E}\right) .
$$

In this case, the continuity of the Nemitsky operator $T_{f}$ is guaranteed by Proposition 2.6. 
In case the open set $E$ has the segment property, the continuity of the operator $T$ was proved in [5].

Proposition 2.6 can be applied, for instance, when there exists a finite partition $\left(E_{i}\right)_{i}$ of $E$ such that for every index $i$, the map $f_{i}$ has a Lip $\cap C^{1}$ extension to an open neighborhood of $E_{i}$. In this case, we can also use the following remark.

Proposition 2.8. Let $f \in \operatorname{Lip}\left(E, \mathbb{R}^{k}\right)$ be a given map, and let $f_{1}, \ldots, f_{S}$ be a finite collection of Lipschitz continuous functions on $\mathbb{R}^{N}$ which induce continuous superposition operators on $W^{1, p}\left(\Omega, \mathbb{R}^{N}\right)$. Assume that for every point $u$ in a dense subset of $E$ there exists an index $i$ with $f(u)=f_{i}(u)$. Then the operator $T_{f}$ is continuous from $W^{1, p}(\Omega, \bar{E})$ into $W^{1, p}\left(\Omega, \mathbb{R}^{k}\right)$.

Proof. Set $E_{i}=\left\{u \in E: f(u)=f_{i}(u)\right\}$, in such a way that the closure of the union of the sets $E_{i}$ covers the closure of $E$. We fix any sequence $\left(u_{n}\right)_{n} \in$ $W^{1, p}(\Omega, \bar{E})$ with $u_{n} \rightarrow u$ in $W^{1, p}\left(\Omega, \mathbb{R}^{N}\right)$ for some map $u \in W^{1, p}(\Omega, \bar{E})$. As was observed in the proof of Proposition 2.6, it suffices to show that, for a subsequence $\left(u_{n}\right)_{n}$, we have

$$
\frac{\partial}{\partial x_{j}}\left(f \circ u_{n}\right)(x) \rightarrow \frac{\partial}{\partial x_{j}}(f \circ u)(x) \quad \text { almost everywhere on } \Omega,
$$

for every coordinate $x_{j}$ in $\mathbb{R}^{n}$. We first observe that we can find a subsequence $\left(u_{n}\right)_{n}$ such that for every $i=1, \ldots, s$ it results

$$
\frac{\partial}{\partial x_{j}}\left(f_{i} \circ u_{n}\right)(x) \rightarrow \frac{\partial}{\partial x_{j}}\left(f_{i} \circ u\right)(x) \quad \text { almost everywhere on } \Omega \text {, }
$$

since the operators $T_{i}$ are continuous on Sobolev spaces by assumption. Since $\bar{E}$ is covered by the union of the sets $\bar{E}_{i}$, Proposition 2.8 can be obtained by the same arguments used in the proof of Proposition 2.6.

\section{SOME EXAMPLES}

The results we have listed in the previous section are quite flexible from the point of view of the applications. In particular, there is a sort of freedom in the choice of the domain $E$ of the map $f$, since the conclusion we want to achieve concerns only the closure of $E$. Hence, if it is more convenient, we can substitute for $E$ any set $E^{\prime}$ which is dense in $E$. This opportunity can be useful in several situations, as in the following example:

Example 3.1. Let $f: \mathbb{R}^{N} \rightarrow \mathbb{R}^{k}$ be a Lipschitz continuous function, and assume that there exists a set $S \subseteq \mathbb{R}^{N}$ such that $f \in C^{1}\left(\mathbb{R}^{N} \backslash S, \mathbb{R}^{k}\right)$ and $f=0$ on $S$. In addition, we assume that, for every $u_{0} \in \partial S$,

$$
\lim _{\substack{u \rightarrow u_{0} \\ u \neq \partial S}} \nabla f(u) \cdot \tau=0 \quad \text { for every } \tau \in T_{\partial S}\left(u_{0}\right) .
$$

Then the operator $T_{f}$ is continuous from $W^{1, p}\left(\Omega, \mathbb{R}^{N}\right)$ into $W^{1, p}\left(\Omega, \mathbb{R}^{k}\right)$. 
Proof. The validity of the conclusion can be checked in many different ways; for instance, we can use Proposition 2.6. To this extent, we define $E$ to be the complement of $\partial S$. From the assumptions we first get that the map $D f$ is continuous on the open set $E \times \mathbb{R}^{N}$. On the other hand, from (2.3) we infer that

$$
D f\left(u_{0}, \tau\right)=0 \quad \text { for every } u_{0} \in \partial S \text { and for every } \tau \in T_{\partial S}\left(u_{0}\right) .
$$

The conclusion easily follows from (3.1) and from the continuity of the map $\tau \rightarrow D f(u, \tau)$ (compare with Lemma 2.3).

We recall that the tangent space to a closed set $S$ reduces to $\{0\}$ in points where $S$ is highly irregular. This means that as $E$ becomes less regular, we have to impose fewer hypotheses on the map $f$.

Example 3.2. Let $f \in \operatorname{Lip}\left(\mathbb{R}^{N}, \mathbb{R}^{k}\right)$ be a given map, and assume that $f$ has continuous first order derivatives outside a singular set $S$ such that $T_{S}(u)=\{0\}$ for every $u \in S$. Then the operator $T_{f}$ is continuous on $W^{1, p}\left(\Omega, \mathbb{R}^{N}\right)$.

By Remark 2.2(iii), the result in Example 3.2 can be applied when the singular set $S$ is discrete. As a first application, we immediately get an easy proof of the fact that the Nemitsky operator associated with the map $z \rightarrow|z|$ is continuous from $W^{1, p}\left(\Omega, \mathbb{R}^{N}\right)$ into $W^{1, p}(\Omega, \mathbb{R})$.

Combining the above remarks with Theorem 0.1 , we can easily exhibit a large number of examples. We limit ourselves to the following result, which can be seen as a corollary of Example 3.2 or of Proposition 2.6, or it can be obtained directly by using Proposition 2.8 :

Example 3.3. Let $g_{1}, \ldots, g_{s}$ be a finite collection of real-valued Lipschitz continuous functions of class $C^{1}\left(\mathbb{R}^{n}, \mathbb{R}\right)$. Then the functions

$$
\operatorname{Max}_{i} g_{i}(\cdot), \operatorname{Min}_{i} g_{i}(\cdot)
$$

induce continuous Nemitsky operators on Sobolev spaces.

Let us now consider as an example the map

$$
f(z)= \begin{cases}\frac{z}{|z|} & \text { if }|z| \geq 1 \\ z & \text { if }|z|<1\end{cases}
$$

In order to prove the continuity of the superposition operator associated with $f$, we can argue in many different ways. For instance, we can apply the arguments in Example 3.1 to the map $f$-Id. Since $f$-Id is identically zero on the unit ball, it suffices to check the validity of (3.1). A second proof can be obtained directly from Proposition 2.8, observing that the restriction of $f$ to the open ball and to its complement have $C^{1}$ extensions to $\mathbb{R}^{N}$.

The same arguments can be applied in order to prove the continuity of the superposition operators associated with the projections on smooth, convex sets. For any closed and convex set $K \subseteq \mathbb{R}^{N}$, we denote by $p_{K}: \mathbb{R}^{N} \rightarrow K$ the projection on $K$. 
Example 3.4. Suppose $K$ is the closure of an open convex set with boundary of class $C^{2}$. Then $p_{K}$ induces continuous Nemitsky operators on Sobolev spaces.

The arguments above lead also to the next example:

Example 3.5. Let $E$ be the union of a finite number of open and convex sets $E_{i}$ with boundary of class $C^{2}$. Then the distance map $d_{E}: \mathbb{R}^{N} \rightarrow \mathbb{R}$ induces continuous Nemitsky operators on Sobolev spaces.

Proof. Let us set $K_{i}=\bar{E}_{i}$. For the proof it suffices to observe that

$$
d_{E}(z)=\operatorname{Min}_{i} d_{K_{i}}(z)=\operatorname{Min}_{i}\left|p_{K_{i}}(z)-z\right| .
$$

By Examples 3.2 and 3.4, the maps $g_{i}(z)=\left|p_{K_{i}}(z)-z\right|$ induce continuous Nemitsky operators on Sobolev spaces. The conclusion easily follows by applying Proposition 2.8.

Actually, we can consider a larger class of convex sets. For example, we can use Proposition 2.6 again in order to prove the continuity of the Nemitsky operator associated with the projection on the convex envelope of a finite number of points. The proof is quite elementary, once one has chosen a convenient partition of $\mathbb{R}^{N}$.

The next corollary could be particularly useful in the applications. Let $M$ be a compact differential submanifold of $\mathbb{R}^{N}$ of class $C^{2}$. Thus, there exists a real number $\varepsilon>0$, and there exist a Lipschitz continuous projection

$$
\pi: \mathscr{N}_{2 \varepsilon}(M)=\left\{z \in \mathbb{R}^{N} \mid d_{M}(z)<2 \varepsilon\right\} \rightarrow M,
$$

defined by

$$
|\pi z-z|=d_{M}(z) .
$$

If $M$ has empty boundary, the projection $\pi$ is continuously differentiable on its domain, and hence it induces continuous maps on Sobolev spaces. Now suppose that $M$ does not have empty boundary; thus $\partial M$ is a differential manifold of class $C^{2}$. In this case, the projection $\pi$ is not everywhere differentiable, and its singular set is precisely the (topological) boundary of

$$
\pi^{-1}(\partial M)=\left\{u \in \mathscr{N}_{2 \varepsilon}(M) \mid \pi u \in \partial M\right\} .
$$

In this case, Proposition 2.6 leads to the following:

Example 3.6. Let $M$ be as above, and let $\Omega \subseteq \mathbb{R}^{n}$ be a given bounded domain. Then for every $\varepsilon$ small enough, the composite map $T u=\pi \circ u$ is continuous from $W^{1, p}\left(\Omega, \overline{\mathcal{N}_{\varepsilon}(M)}\right)$ into $W^{1, p}(\Omega, M)$.

Proof. If $\varepsilon$ is small enough, the map

$$
\begin{gathered}
\pi_{\partial}: \mathscr{N}_{2 \varepsilon}(\partial M)=\left\{z \in \mathbb{R}^{N} \mid d_{\partial M}(z)<2 \varepsilon\right\} \rightarrow \partial M, \\
\left|\pi_{\partial} z-z\right|=d_{\partial M}(z),
\end{gathered}
$$


is well defined on $\mathscr{N}_{2 \varepsilon}(\partial M)$. Moreover, it has continuous and bounded first order partial derivatives on its domain. We set

$$
E_{1}=\left\{z \in \mathscr{N}_{\varepsilon}(M) \mid \pi z \in M \backslash \partial M\right\},
$$

and

$$
E_{2}=\text { interior of }\left\{z \in \mathscr{N}_{\varepsilon}(M) \mid \pi z=\pi_{\partial} z \in M\right\} .
$$

Since $\left.\pi\right|_{E_{2}}=\pi_{\partial}$, and $\left.\pi\right|_{E_{1}}$ has a $C^{1}$ extension to a neighborhood of $E_{1}$ by our smoothness assumptions on $M$, Proposition 2.6 applies immediately.

\section{ACKNOWLEDGMENT}

I would like to thank Professor Gianni Dal Maso for useful and stimulating discussions on this problem.

\section{REFERENCES}

1. L. Ambrosio and G. Dal Maso, A general chain rule for distributional derivatives, Proc. Amer. Math. Soc. 108 (1990), 691-702.

2. J. P. Aubin and I. Ekeland, Applied nonlinear analysis, John Wiley \& Sons, New York, 1984.

3. L. Boccardo and F. Murat, Remarques sur l'homogeineisation de certains problemes quasilineaires, Portugal Math. 41 (1982), 535-562.

4. G. Buttazzo and A. Leaci, A continuity theorem for operators from $W^{1, q}(\Omega)$ into $L^{q}(\Omega)$, J. Funct. Anal. 58 (1984), 216-224.

5. G. Dal Maso and R. Musina, An approach to the thin obstacle problem for variational functions depending on vector valued functions, Comm. Partial Differential Equations 14 (1989), 1717-1743.

6. H. Federer, Geometric measure theory, Springer, Berlin, 1969.

7. M. Marcus and V. J. Mizel, Absolute continuity on traks and mappings of Sobolev spaces, Arch. Rational Mech. Anal. 45 (1972), 294-320.

8. __ Every superposition operator mapping one Sobolev space into another is continuous, J. Funct. Anal. 33 (1979), 217-229.

9. C. B. Morrey, Multiple integrals in the calculus of variations, Springer, Berlin, 1966.

10. K. Steffen, Isoperimetric inequalities and the problem of plateau, Math. Ann. 222 (1976), 97-144.

International Centre for Theoretical Physics, Trieste, Italy 